\title{
Trajectory Design for the Phobos and Deimos \& Mars Environment Spacecraft
}

\author{
Anthony L. Genova ${ }^{1}$ and David. J. Korsmeyer ${ }^{2}$ \\ NASA Ames Research Center (ARC), Moffett Field, CA, 94035 \\ Michel E. Loucks ${ }^{3}$ \\ Space Exploration Engineering Corporation, Friday Harbor, WA 98250 \\ Fan Yang Yang ${ }^{4}$ \\ Millennium Engineering \& Integration Co., based at NASA ARC, Moffett Field, CA 94035 \\ and \\ Pascal Lee ${ }^{5}$ \\ SETI Institute, Mountain View, CA 94035 \& Mars Institute, Moffett Field, CA 94035
}

\begin{abstract}
The presented trajectory design and analysis was performed for the Phobos and Deimos \& Mars Environment (PADME) mission concept as part of a NASA proposal submission managed by NASA Ames Research Center in the 2014-2015 timeframe. The PADME spacecraft would be a derivative of the successfully flown Lunar Atmosphere \& Dust Environment Explorer (LADEE) spacecraft. While LADEE was designed to enter low-lunar orbit, the PADME spacecraft would instead enter an elliptical Mars orbit of 2-week period. This Mars orbit would pass by Phobos near periapsis on successive orbits and then raise periapsis to yield close approaches of Deimos every orbit thereafter.
\end{abstract}

\section{Introduction}

$\mathrm{T}$ his paper presents a trajectory design capable of placing the Phobos and Deimos \& Mars Environment (PADME) spacecraft ${ }^{1,2}$ in an elliptical Mars orbit with successive low-altitude (2 to $\left.10 \mathrm{~km}\right)$ passes/flybys of Phobos and Deimos and with relatively low flyby speeds. A similar trajectory design was presented by Tolson et al. ${ }^{3}$ in 1976 and by Diehl et al. ${ }^{4}$ in 1978, both with regard to an extension option for the Viking 1 mission; the Viking 1 spacecraft performed 14 flybys of Phobos within an altitude range of $89-213 \mathrm{~km}$, which yielded mass and density measurements of Phobos 5 . ALADDIN proposed an interesting trajectory that would performs flybys of Phobos and Deimos $^{6}$, although this trajectory is not compatible with the PADME mission (discussed later).

For about the same amount of $\triangle \mathrm{V}$ needed by the LADEE spacecraft to enter low-lunar orbit (LLO), the PADME spacecraft is able to enter an elliptical Mars orbit with $\approx 2$-week period. This 2-week cadence allows for operational flexibility and planning between flyby (science) events, and the ability to expend little $\Delta \mathrm{V}$ to raise periapsis to (near) Deimos distance to begin the Deimos cycler orbit phase. By adjusting the apoapsis altitude of the elliptical Mars orbit, resonance with Phobos and Deimos is achieved, with a total of 16 Phobos and 9 Deimos flybys yielded by the trajectory designed for the PADME spacecraft, with details presented herein.

\footnotetext{
${ }^{1}$ Trajectory Designer, Mission Design Division, NASA ARC, Moffett Field, CA 94035, AIAA Member

${ }^{2}$ Director of Engineering, Engineering Directorate, NASA ARC, Moffett Field, CA 94035, AIAA Associate Fellow

${ }^{3}$ Chief Astrodynamics Scientist, Space Exploration Engineering Corporation, Friday Harbor, WA 98250

${ }^{4}$ Mission Design Engineer, Mission Millennium Engineering \& Int., Co., NASA ARC, Moffett Field, CA 94035

${ }^{5}$ Chief Scientist, SETI Institute, Mountain View, CA 94035 \& Mars Institute, Moffett Field, CA 94035
} 


\section{Assumptions \& Constraints}

There were several requirements and constraints that significantly influenced the trajectory design for the PADME spacecraft, as summarized in Table 1 . The $\Delta \mathrm{V}$ requirement (No. 2, Table 1), constrains the minimum orbit energy of the captured Mars orbit, while science-related requirements significantly influence the Phobos and Deimos flyby strategy.

The primary software tool used for the presented trajectory design and analysis was STK/Astrogator, which utilized an $8^{\text {th }} / 9^{\text {th }}$ order Runge-Kutta numerical integrator for orbit propagation within a force model that included an N-body gravity field (Sun, Earth, Moon, Mars modeled as gravity fields with the remaining major planets modeled as point masses), thermal and solar radiation pressure, and atmosphere models at both Earth and Mars. The best available shape models were utilized for both Phobos and Deimos (from Gaskell and Thomas, respectively) given the close-approaches designed into the presented trajectory.

Table 1. Description of Trajectory-Influenced Requirements and Constraints

\begin{tabular}{|c|c|c|}
\hline $\begin{array}{l}\text { Require- } \\
\text { ment } \\
\text { Number }\end{array}$ & Description of Requirement or Constraint & $\begin{array}{l}\text { Requirement } \\
\text { Imposed by }\end{array}$ \\
\hline 1 & $\begin{array}{l}\text { PADME Spacecraft will launch as a primary payload } \\
\text { from NASA Kennedy Space Center (KSC) }\end{array}$ & LAUNCH \\
\hline 2 & $1,205 \mathrm{~m} / \mathrm{s}$ Maximum $\Delta \mathrm{V}$ capability of the spacecraft & PROPULSION \\
\hline 3 & Enter a Mars orbit in resonance with Phobos and Deimos & SCIENCE \\
\hline 4 & $\begin{array}{l}\text { Target resonance trajectory to yield low altitude passes of } \\
\text { Phobos and Deimos (between } 2 \text { and } 10 \mathrm{~km} \text { ) }\end{array}$ & $\begin{array}{c}\text { SCIENCE } \\
\& \text { NAVIGATION }\end{array}$ \\
\hline 5 & $\begin{array}{l}\text { At least eight Phobos and eight Deimos flybys must occur } \\
\text { with a speed }<1.5 \mathrm{~km} / \mathrm{s}\end{array}$ & $\begin{array}{c}\text { NEUTRON } \\
\text { SPECTROMETER }\end{array}$ \\
\hline 6 & $\begin{array}{l}\text { At least two Phobos flybys must occur with a close-approach } \\
\text { speed }>2 \mathrm{~km} / \mathrm{s} \text { (for Deimos, }>1 \mathrm{~km} / \mathrm{s} \text { ) }\end{array}$ & DUST DETECTION \\
\hline 7 & $\begin{array}{l}\text { All but one Phobos flyby must have the spacecraft close- } \\
\text { approach (nadir projection onto Phobos' surface) occur in } \\
\text { direct sunlight }\end{array}$ & LIGHTING \\
\hline 8 & $\begin{array}{l}\text { Exactly one Phobos flyby must have the spacecraft close- } \\
\text { approach (nadir projection onto Phobos' surface) occur on the } \\
\text { terminator }\end{array}$ & LIGHTING \\
\hline 9 & $\begin{array}{l}\text { All but three Deimos flybys must have the spacecraft close- } \\
\text { approach (nadir projection onto Phobos' surface) occur in } \\
\text { direct sunlight }\end{array}$ & LIGHTING \\
\hline 10 & $\begin{array}{l}\text { Exactly three Deimos flybys must have the spacecraft close- } \\
\text { approach (nadir projection onto Phobos' surface) occur on the } \\
\text { terminator }\end{array}$ & LIGHTING \\
\hline 11 & $\begin{array}{l}\text { Maintain direct line-of-sight with the Earth during every } \\
\text { close-approach of both Phobos and Deimos }\end{array}$ & COMMUNICATIONS \\
\hline 12 & $\begin{array}{l}\text { Establish opportunity for viewing (within } 50 \mathrm{~km} \text { ) of pre- } \\
\text { determined target on a sunlit equatorial region of Phobos at } \\
\text { multiple positions (at least } 3 \text { ) along its orbit with true anomaly } \\
\text { span }>45 \mathrm{deg}\end{array}$ & LIBRATION SCIENCE \\
\hline 13 & $\begin{array}{l}\text { Equally balance the latitude/longitude of the close-approaches } \\
\text { for both Phobos and Deimos }\end{array}$ & GRAVITY SCIENCE \\
\hline
\end{tabular}




\section{Trajectory Design}

The following section outlines the trajectory design, mission extension options, and future launch opportunities. Libration measurements of Phobos' orbit and gravity field measurements of both Martian moons are also discussed.

\section{A. General Trajectory Design}

The presented trajectory assumes a launch on August 3, 2020, the opening of the optimal 21-day launch period. Both Phobos and Deimos cycler orbit phases are shown together in Fig. 1 (left), which also shows a zoomed in image near periapsis to focus on science events (bottom right) and a Phobos inertial view that shows all of the relevant flyby paths.

It can be seen from Fig. 1 that after the initial Mars-orbit insertion, the Mars periapsis is incrementally raised to encounter Phobos off-periapsis (Fig. 1, A), which allows the chance for a relatively large range of libration measurements of Phobos' orbit and gravity measurements of the moon's gravity field (when combined with future measurements).

After seven such Phobos encounters at $2.27 \mathrm{~km} / \mathrm{s}$ flyby speed (including a warm-up encounter at $10 \mathrm{~km}$ flyby altitude), periapsis is raised to obtain more diverse libration and gravity science field measurements, now at lower flyby speeds of $1.45 \mathrm{~km} / \mathrm{s}$ (Fig. 1, B). After four such flybys, periapsis is raised once again to increase the quality of the science data set, with five Phobos passes at 1 $\mathrm{km} / \mathrm{s}$ (three flybys before a solar exclusion zone blackout window and two afterward, Fig. 1, C). A summary table of the selected flyby sequence is shown in Table 2, where it is seen that all Table 1 requirements are met.

The largest maneuver to enter the required Phobos/Deimos resonance orbit is the Mars orbit insertion, which required $649 \mathrm{~m} / \mathrm{s}$ (highest injection $\Delta \mathrm{V}$ requirement throughout the 21-day launch period). This injection maneuver was fine-tuned to vary the orbit period to yield the required Phobos/Deimos resonance orbit with 2-week period (apoapsis altitude of $\sim 230,000 \mathrm{~km}$ ). The second largest maneuver was calculated as $59 \mathrm{~m} / \mathrm{s}$ to "switch gears" from the
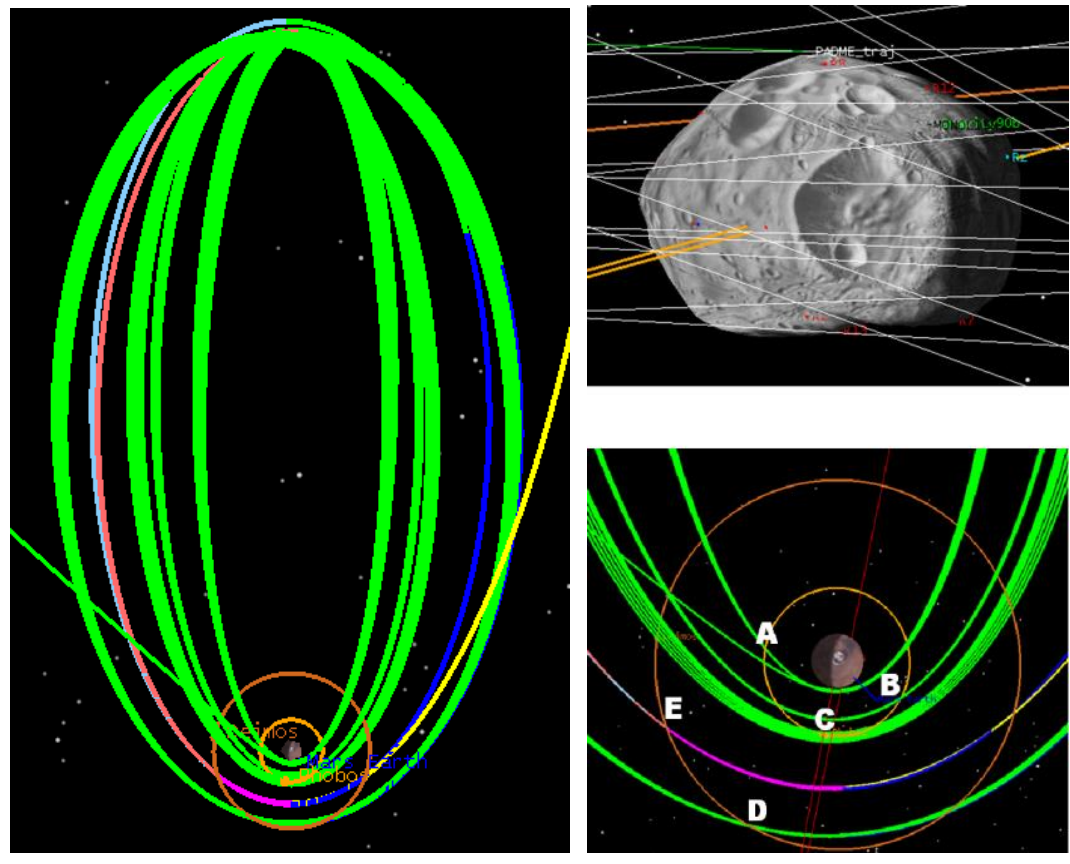

Figure 1. Phobos \& Deimos Cycler Orbit Sequence. Trajectory shown in the Mars inertial frame (left and bottom-right images) and in the Phobos inertial frame (top-right image).

Phobos to Deimos cycler orbit, where nine Deimos flybys are planned at two Mars periapsis altitudes (Fig. 1, D and E). It is of note that the ALADDIN concept did not require a "gear-switch", as their orbit was designed to pass by both Martian moons during each orbit, although at the expense of a significant increase in the flyby speed of Deimos; the same trajectory can be used for opportunities with high arrival declinations at Mars (discussed later). Given the relatively low $\Delta \mathrm{V}$ requirement to reach the first Phobos flyby, the Mars periapsis altitude was increased to improve the readings from the neutron spectrometer data.

Varying the Mars periapsis to meet the libration science requirements for the Phobos cycler sequence totaled 58 $\mathrm{m} / \mathrm{s}$, with the additional $\Delta \mathrm{V}$ dedicated to cycler orbit maintenance (approximately $2 \mathrm{~m} / \mathrm{s}$ per Phobos or Deimos cycle/flyby, assuming a fixed Mars periapsis altitude) and $58 \mathrm{~m} / \mathrm{s}$ for the Mars-escape disposal maneuver. This yields a total $\Delta \mathrm{V}$ requirement of $935 \mathrm{~m} / \mathrm{s}$ which meets the $1,205 \mathrm{~m} / \mathrm{s}$ total $\Delta \mathrm{V}$ constraint and allows $270 \mathrm{~m} / \mathrm{s}(22.4 \%)$ for navigation, margin, extension mission opportunities, etc. It is of note that direct transfers for future Mars launch periods in 2022 and 2024 were analyzed as backup opportunities and were both shown to be compatible with the presented PADME mission concept. The total $\Delta \mathrm{V}$ requirement calculated for these launch periods was less than the maximum-allocated $1,205 \mathrm{~m} / \mathrm{s}$ (discussed in a later section). 
Table 2. Summary of Phobos and Deimos Flyby Characteristics

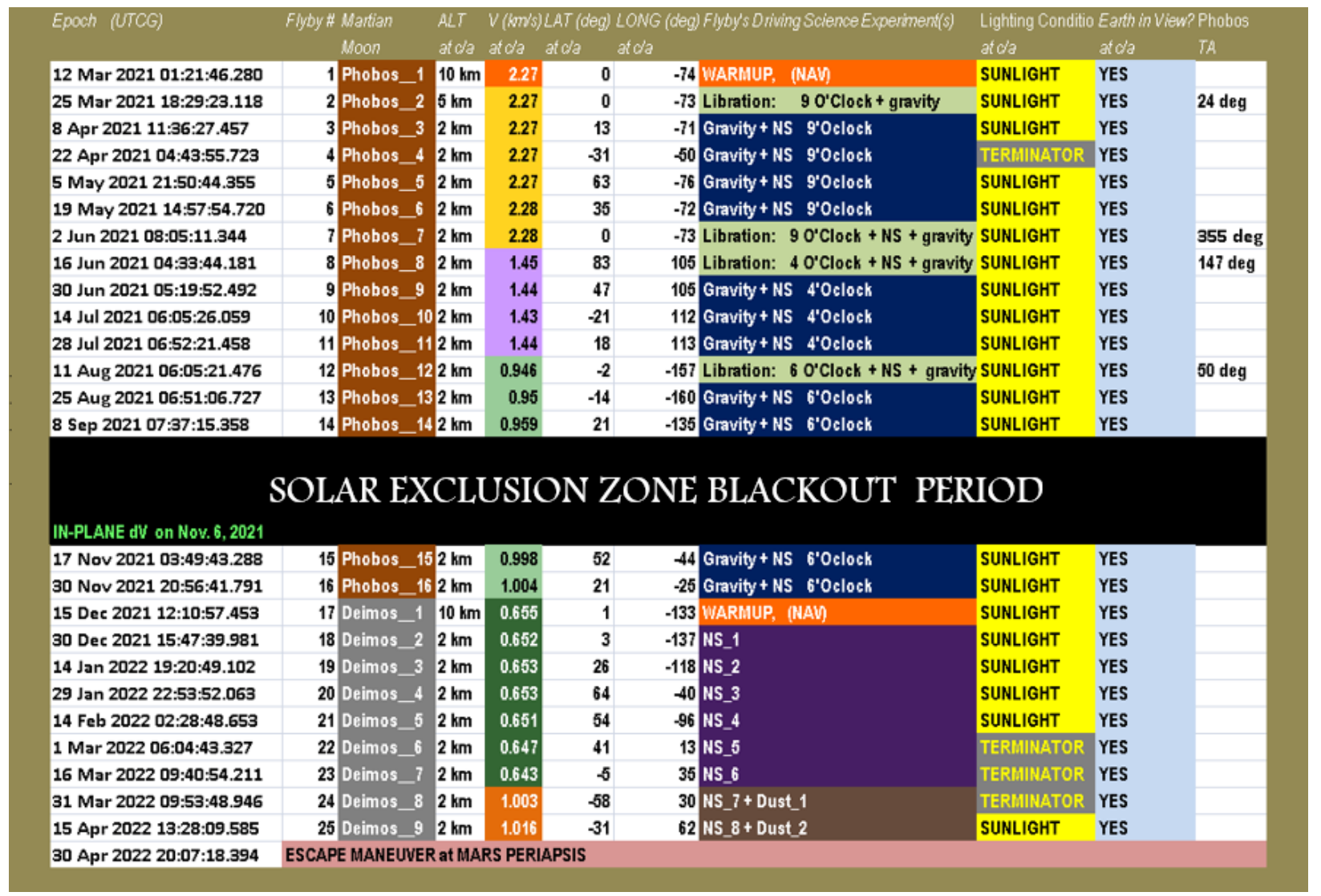

\section{B. Libration Science Measurements of Phobos' Orbit}

Four Phobos flybys are specified as providing opportunities for libration science; however, libration imaging can occur on any flyby that passes within $50 \mathrm{~km}$ of a sunlit region of Phobos' surface. Three of the four specified libration flybys allow for two surface regions separated by more than 50 degrees of longitude along the equator to be imaged, which is not required but significantly helps improve science quality and allows redundancy in meeting the libration science requirement consisting of at least 3 measurements of the same equatorial surface region spanning greater than 45 degrees in true anomaly of Phobos' orbit. Given Phobos' rotation property of being tidally locked with Mars, the trajectory design varies the Mars periapsis to allow for different arrival geometries (i.e., varying B planes) at Phobos.

The second flyby from Table 2 yields the $1^{\text {st }}$ libration measurement located at 0 degrees latitude and 160 degrees west longitude on the surface of Phobos (Fig. 2). Given the $2.27 \mathrm{~km} / \mathrm{s}$ Phobos flyby speed at this time in the sequence, the spacecraft spends 21 -seconds within $50 \mathrm{~km}$ (and within view) of this targeted surface location (Fig. 3). On this same flyby, the spacecraft also images another location on Phobos' surface so as to yield two acceptable sets of libration measurements. This second equatorial imaging location is located at 108 degrees west longitude $(0$, 108). The PADME spacecraft would spend a total of 27 seconds within $50 \mathrm{~km}$ of $(0,-108)$, with a close-approach altitude of $6.5 \mathrm{~km}$. The true anomaly at this Phobos position is 24 degrees (Fig. 1, A). 
After seven such Phobos encounters at $2.27 \mathrm{~km} / \mathrm{s}$ flyby speed (including a warm-up encounter at $10 \mathrm{~km}$ flyby altitude), The largest maneuver to enter the required Phobos/Deimos resonance orbit is the Mars orbit insertion, which required $649 \mathrm{~m} / \mathrm{s}$; this corresponded to the highest $\Delta \mathrm{V}$ requirement throughout the optimized 21-day launch period associated with a launch from NASA Kennedy Space Center. The second largest maneuver was calculated as $59 \mathrm{~m} / \mathrm{s}$ to "switch gears" from the Phobos to Deimos cycler orbit, where nine Deimos flybys are planned at two Mars periapsis altitudes (Fig. 1, D and E). It is of note that the ALADDIN concept did not require a "gear-switch", as its orbit was designed to pass by both Martian moons with a fixed Mars periapsis. However, such an orbit yields a significantly higher flyby speed of Deimos; still, there are applications relevant to the PADME spacecraft when the arrival declination upon Mars arrival is relatively high (discussed in the "Future Launch Opportunities to Mars" subsection). Given the relatively low $\Delta \mathrm{V}$ requirement to reach the first Phobos flyby, the Mars periapsis altitude was increased to improve the readings from the neutron spectrometer data.

The second flyby specified for Phobos libration science corresponds to the $7^{\text {th }}$ Phobos flyby seen in Table 2 . The flyby speed is $2.28 \mathrm{~km} / \mathrm{s}$ which yields a 21 -second viewing opportunity within $50 \mathrm{~km}$ of the $(0,-160)$ libration target; the close-approach altitude above $(0$, 160) was calculated as $10.9 \mathrm{~km}$. For the $(0,-108)$ libration target, more time (26 seconds of total time) is spent within $50 \mathrm{~km}$; the spacecraft reaches its closeapproach altitude of $3.4 \mathrm{~km}$ and is then able to continue viewing ( 0 , 108) on its outbound trajectory. Phobos' true anomaly for this flyby is 355 degrees given 29 degrees of natural precession since the second flyby (Fig. 1, A).

During the third libration-
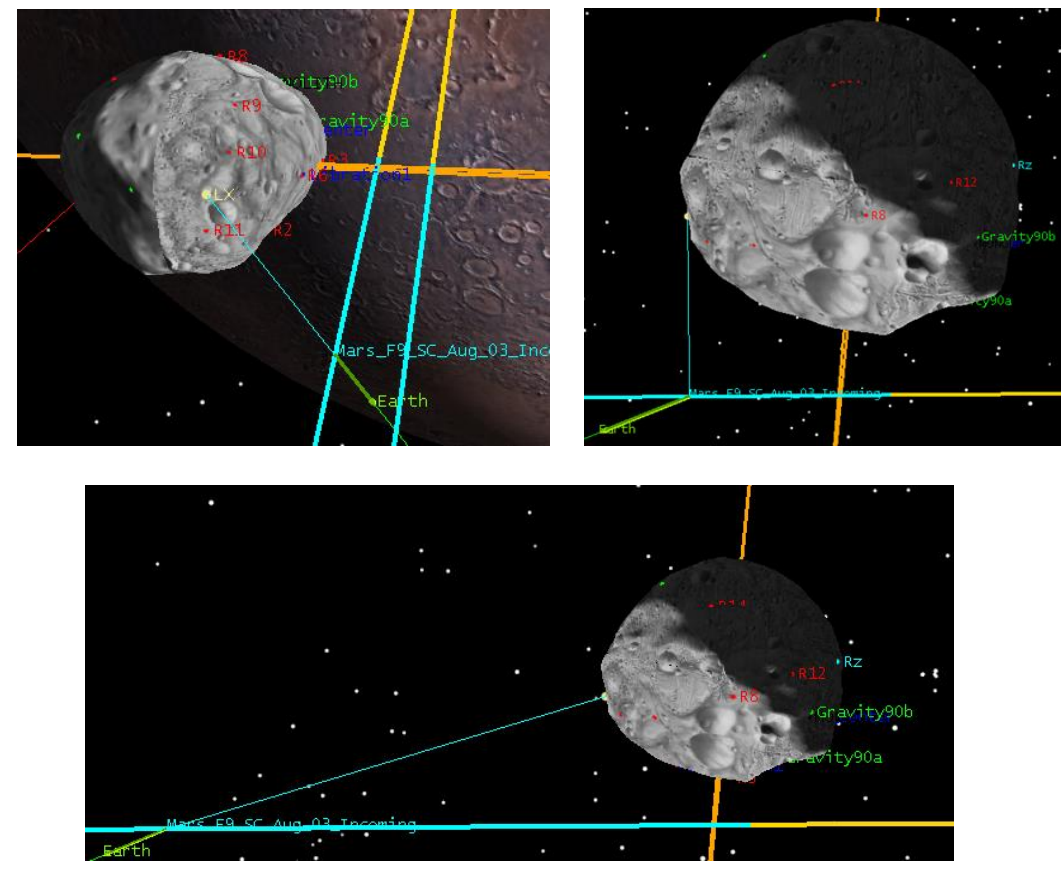

Figure 2. Libration Measurement for $2^{\text {nd }}$ Phobos Flyby. Surface Target chosen for imaging on this flyby: (0 degrees, 160 degrees west).

specified Phobos flyby (this is the $8^{\text {th }}$ Phobos flyby per Table 2$)$, the $(0,-108)$ libration target is in shadow, thus no viewing opportunity of this target is possible during this flyby. However, the $(0,-160)$ libration target is in direct sunlight and provides an important opportunity to acquire a libration measurement on the outbound of the trajectory (i.e., after reaching Mars periapsis: Fig. 1, B). This opportunity is important, since it significantly increases the range of sampled Phobos' true anomaly values by adding 147 degrees to the data set for the $(0,-160)$ target. As the flyby speed is reduced to $1.45 \mathrm{~km} / \mathrm{s}$, the viewing opportunity window is longer (compared to the previous libration measurements), with a total of 32 seconds of viewing time spent within $50 \mathrm{~km}$ of $(0,-160)$. The close-approach altitude above $(0,-160)$ was calculated as $13 \mathrm{~km}$.

The fourth specified libration flyby is the $12^{\text {th }}$ Phobos flyby. The associated flyby speed is the lowest among any specified libration flybys, at $0.946 \mathrm{~km} / \mathrm{s}$ since it occurs closest to Mars periapsis (Fig. 1, C). The $(0,-160)$ libration target is within $50 \mathrm{~km}$ of the PADME spacecraft for 65 -seconds, the longest viewing opportunity calculated. The close-approach altitude above $(0,-160)$ was calculated as $2.1 \mathrm{~km}$. The $(0,-108)$ libration target is also imaged on this flyby at $0.946 \mathrm{~km} / \mathrm{s}$ speed within a 58 -second viewing window within $50 \mathrm{~km}$ of this libration target. The closeapproach altitude above $(0,-108)$ was calculated as $7.8 \mathrm{~km}$. The true anomaly of Phobos' orbit was 50 degrees for this flyby.

A summary of the libration science measurements are listed below; it can be seen in Table 3 that the 45 -degree range in Phobos true anomaly values sampled (at least 3 measurements) is met for both libration targets despite only one such target needed to meet the relevant science requirement. Specifically, a 152-degree total true anomaly range is acquired for $(0,-160)$ and a 55-degree range for $(0,-108)$. 


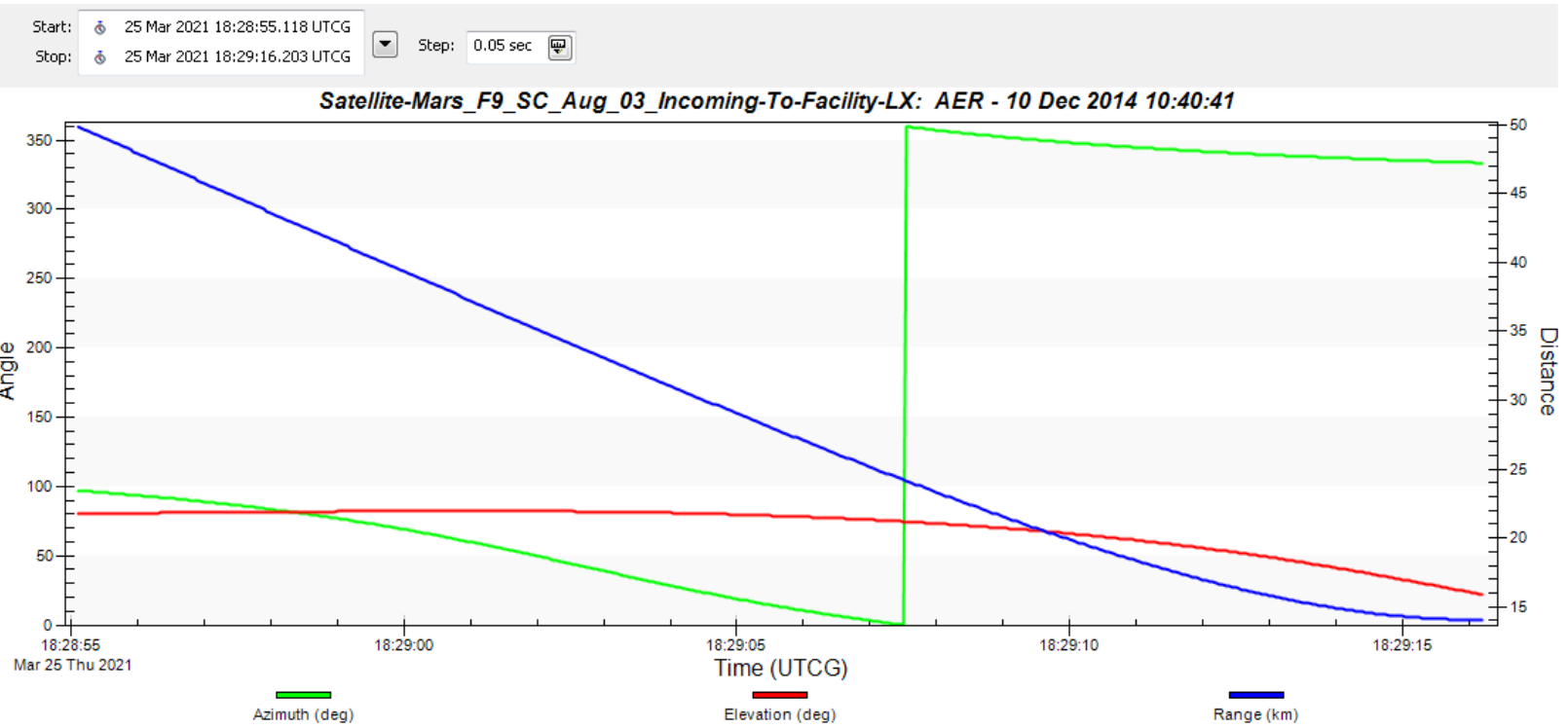

Figure 3. Libration Measurement for $2^{\text {nd }}$ Phobos Flyby. Surface Target chosen for imaging on this flyby: $(0$ degrees, 160 degrees west).

Table 3. Summary of Phobos Libration Science Measurements.

\begin{tabular}{|c|c|c|c|}
\hline $\begin{array}{c}\text { Libration Surface } \\
\text { Target Coordinates } \\
\text { (latitude, longitude) }\end{array}$ & $\begin{array}{c}\text { Flyby Number } \\
\text { from which } \\
\text { Measurement is } \\
\text { acquired }\end{array}$ & $\begin{array}{c}\text { True Anomaly } \\
\text { of Phobos' Orbit } \\
\text { at Time of Measurement }\end{array}$ & $\begin{array}{c}\text { Delta True Anomaly } \\
\text { of Phobos' Orbit } \\
\text { Cumulative values shown } \\
\text { for both targets) }\end{array}$ \\
\hline$(0,-160)$ & Flyby \#2 & 24 degrees & 0 degrees \\
\hline$(0,-160)$ & Flyby \#7 & 355 degrees & 29 degrees \\
\hline$(0,-160)$ & Flyby \#8 & 147 degrees & 152 degrees \\
\hline$(0,-160)$ & Flyby \#12 & 50 degrees & 0 degrees \\
\hline$(0,-108)$ & Flyby \#2 & 24 degrees & 29 degrees \\
\hline$(0,-108)$ & Flyby \#7 & 355 degrees & 55 degrees \\
\hline$(0,-108)$ & Flyby \#12 & 50 degrees & 152 degrees \\
\hline
\end{tabular}




\section{Gravity Science Measurements of Phobos and Deimos}

The PADME spacecraft is equipped with a neutron spectrometer that has the ability to obtain mass measurements from both Phobos and Deimos to better understand the gravity field of both Martian moons.

For each gravity science flyby, the coordinates of closeapproaches of both Phobos and Deimos by the spacecraft are plotted, with an inertial view of all (nine) Deimos flybys displayed as well (Figs. 4, 5 and 6).

Given the lighting and other constraints, the latitude and longitude of closeapproach was balanced as best as possible throughout the Phobos/Deimos cycler orbit sequence. The libration science requirement (No. 12 in Table 1) is coupled with the gravity science requirement (No. 13 in Table 1) since sampling of the Martian moons at different true anomalies given the tidal lock of both moons. Since both libration and gravity science can occur in the same flyby, the coupling of these requirements is met with a single trajectory design feature, namely that of varying the Mars periapsis altitude to yield the necessary measurements at varying true anomalies. The gravity science data shown in Figs. 16 and 17 were deemed sufficient to meet Requirement number 13 in Table 1.

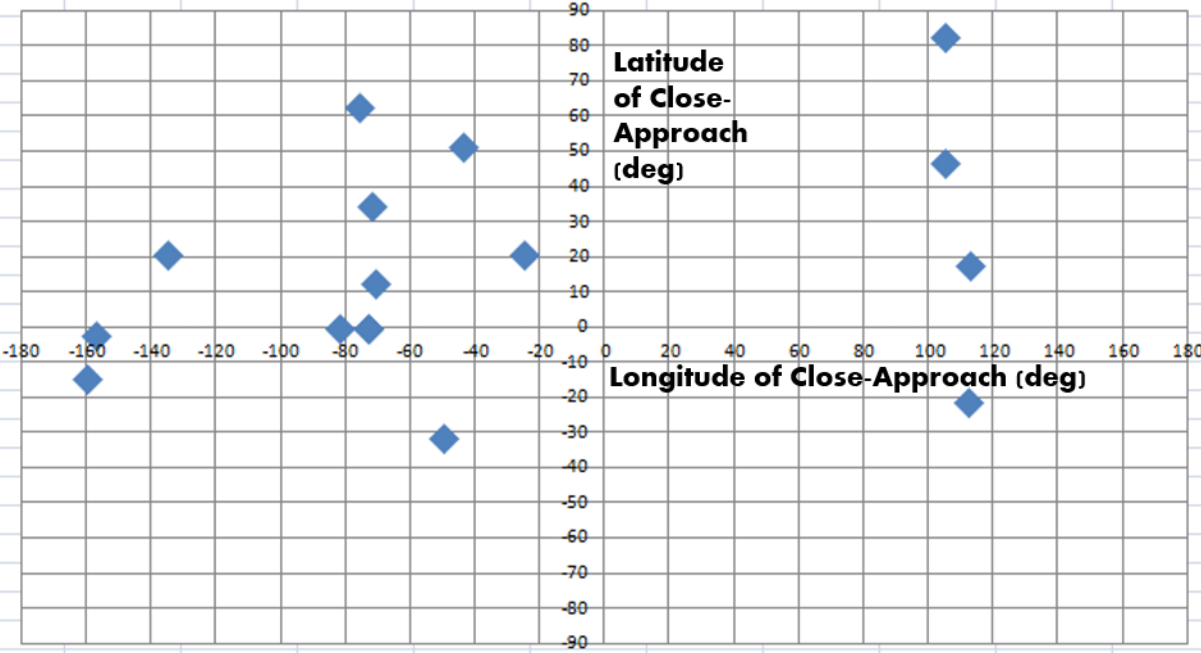

Figure 4. Phobos Gravity Science Measurements. Latitude \& Latitude of closeapproach for Phobos flybys ( $1^{\text {st }}$ warmup flyby of Phobos not shown); X-axis: Longitude, Y-axis: Latitude

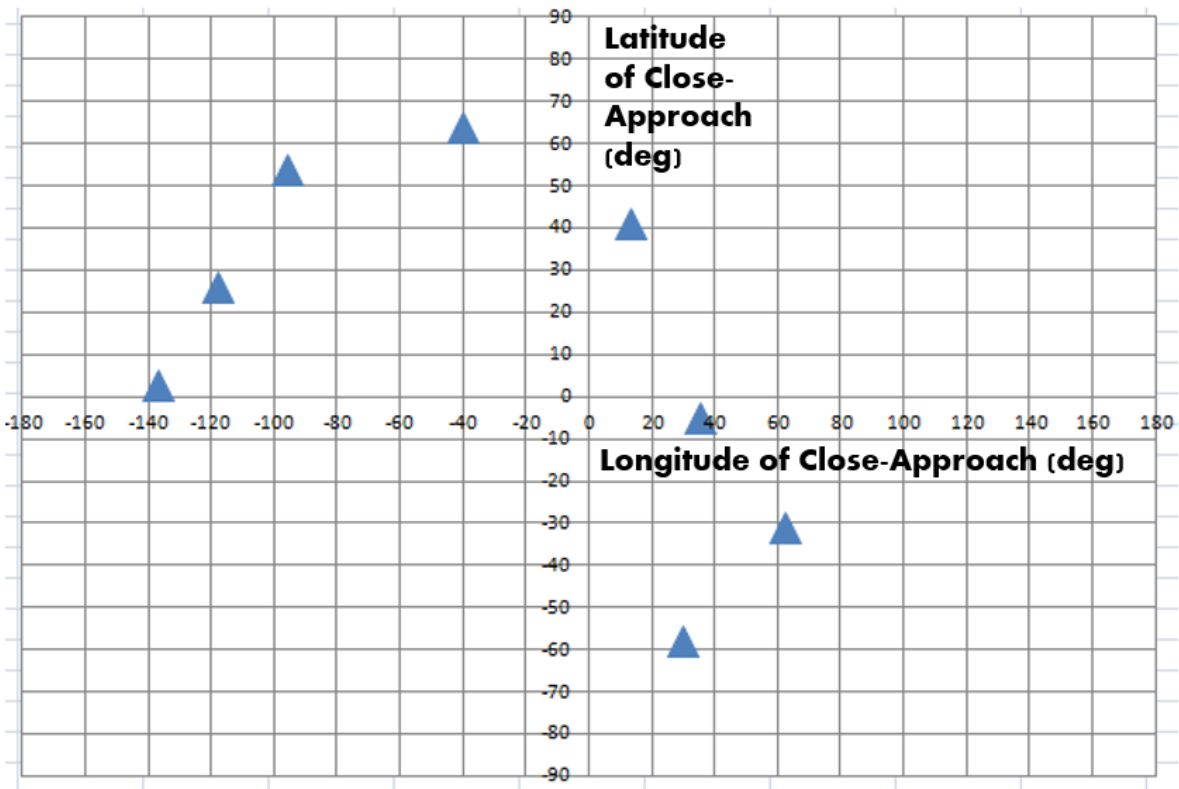

Figure 5. Deimos Gravity Science Measurements. Latitude \& Latitude of closeapproach for Deimos flybys ( $1^{\text {st }}$ warmup flyby of Deimos not shown); X-axis: Longitude, Y-axis: Latitude 

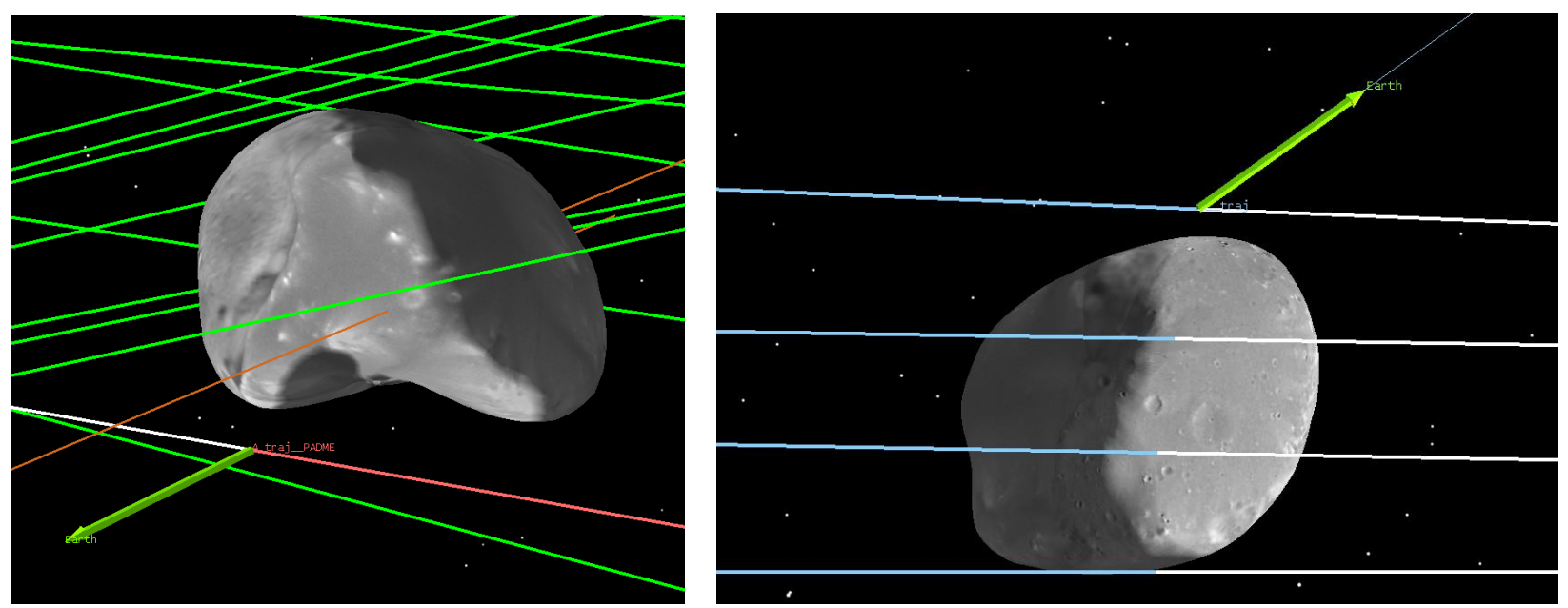

Figure 6. Deimos Flyby Phase: flybys are shown in separate Deimos-centric inertial frames (left: all flybys shown with edge-on view of the Deimos equatorial plane; right: four flybys shown from view onto the north pole, with better view of the terminator per Req. No. 10 in Table 1).

\section{Disposal \& Mission Extension Options}

After the ninth and final flyby of Deimos, the disposal plan for the Table 4. Top 8 Asteroid Rendezvous Opportunities for the PADME Spacecraft, PADME spacecraft is that of heliocentric orbit that does not impact Mars for at least 100 years. To reach this heliocentric disposal orbit, the spacecraft would perform an escape maneuver at Mars periapsis 2 weeks after the final Deimos flyby; the $\Delta \mathrm{V}$ required for this maneuver was calculated as $58 \mathrm{~m} / \mathrm{s}$.

Alternatively, the Mars-escape maneuver can be increased and/or delayed to allow for alignment with an interplanetary target of opportunities are sorted by $\triangle V$ required for trans-NEO injection from Mars and the $N E O$ rendezvous maneuver.

\begin{tabular}{|c|c|c|}
\hline $\begin{array}{c}\text { Opportunity } \\
\text { Number }\end{array}$ & $\begin{array}{c}\text { Asteroid Number } \\
\text { (Designation) }\end{array}$ & $\begin{array}{c}\Delta \text { V Required for } \\
\text { Rendezvous }\end{array}$ \\
\hline 1 & $68278(2001 \mathrm{FC} 7)$ & $1.14 \mathrm{~km} / \mathrm{s}$ \\
\hline 2 & $96631(1999 \mathrm{FP} 59)$ & $2.67 \mathrm{~km} / \mathrm{s}$ \\
\hline 3 & $256004(2006 \mathrm{UP})$ & $3.11 \mathrm{~km} / \mathrm{s}$ \\
\hline 4 & $162783(2000 \mathrm{YJ} 11)$ & $3.75 \mathrm{~km} / \mathrm{s}$ \\
\hline 5 & 65803 Didymos (1996 GT) & $4.69 \mathrm{~km} / \mathrm{s}$ \\
\hline 6 & $363305(2002 \mathrm{NV} 16)$ & $4.78 \mathrm{~km} / \mathrm{s}$ \\
\hline 7 & $173664(2001 \mathrm{JU} 2)$ & $4.82 \mathrm{~km} / \mathrm{s}$ \\
\hline 8 & $302871(2003 \mathrm{HA} 22)$ & $4.98 \mathrm{~km} / \mathrm{s}$ \\
\hline
\end{tabular}
interest. For purposes of better understanding such mission extension options, both flyby and rendezvous trajectory opportunities to nearEarth objects (NEOs) and Mars-crossing asteroids (MCAs) were calculated using a 2-body force model.

The top 8 asteroid rendezvous opportunities are listed in Table 4, where it can be seen that no opportunity requires less than $1 \mathrm{~km} / \mathrm{s}$ (many require significantly higher $\Delta \mathrm{V}$ requirements). Given the 1,205 $\Delta \mathrm{V}$ requirement imposed by the propulsion system (No. 2, Table 1), the PADME spacecraft can afford to expend about $250 \mathrm{~m} / \mathrm{s}$, including the disposal/escape maneuver, for an extension mission option. Thus a rendezvous mission was deemed infeasible for the analyzed launch opportunity. 
Mission extension options that entail an asteroid flyby require far less $\Delta \mathrm{V}$ than the aforementioned rendezvous opportunities. The top 10 NEO flyby opportunities are listed in Table 5; the top 3 opportunities require an injection/escape $\Delta \mathrm{V}$ less than $250 \mathrm{~m} / \mathrm{s}$ and are thus feasible options for the PADME spacecraft. The lowest $\Delta \mathrm{V}$ requirement is associated with the flyby opportunity of NEO 85585 Mjolnir (1998 FG2) which has an estimated diameter of 127 to 284 meters and represents the axe-hammer of the mythological Norse god Thor. A high-fidelity trajectory to Mjolnir was thus calculated and a view of the trajectory at time of NEO closeapproach is displayed in Fig. 7. In this trajectory, the PADME spacecraft would wait in Mars orbit until August 3, 2023 (allowing time for more Deimos and/or Phobos flybys) which is when the trans-NEO injection (TNI) maneuver is performed at Mars periapsis. The TNI is the only deterministic maneuver required (including disposal) with a $\Delta \mathrm{V}$ of $67 \mathrm{~m} / \mathrm{s}$ calculated via the high-fidelity STK/Astrogator model mentioned in the Assumptions \& Constraints section. The flyby of Mjolnir would occur almost 9 months later on April 24, 2024. The close-approach of the flyby can be varied, but was chosen as $1 \mathrm{~km}$ for the presented case; the flyby speed of Mjolnir was calculated as 9.5 $\mathrm{km} / \mathrm{s}$. After the flyby, the spacecraft would be in a heliocentric disposal orbit without the need to perform a maneuver. The total $\Delta \mathrm{V}$ requirement yielded was $944 \mathrm{~m} / \mathrm{s}$, well within the $1,205 \mathrm{~m} / \mathrm{s}$ limit.

Table 5. Top 10 NEO Flyby Opportunities for the PADME Spacecraft. Opportunities are sorted by $\triangle V$ required for trans-NEO injection from Mars.

\begin{tabular}{|c|c|c|}
\hline $\begin{array}{c}\text { Opportunity } \\
\text { Number }\end{array}$ & NEO Number (Designation) & $\begin{array}{c}\Delta \text { V Required for } \\
\text { Flyby }\end{array}$ \\
\hline 1 & 85585 Mjolnir (1998 FG2) & $75 \mathrm{~m} / \mathrm{s}$ \\
\hline 2 & $252558(2001$ WT1) & $131 \mathrm{~m} / \mathrm{s}$ \\
\hline 3 & $203217(2001$ FX9) & $227 \mathrm{~m} / \mathrm{s}$ \\
\hline 4 & $405212(2003$ QC10) & $288 \mathrm{~m} / \mathrm{s}$ \\
\hline 5 & $276468(2003$ HQ32) & $306 \mathrm{~m} / \mathrm{s}$ \\
\hline 6 & $68278(2001$ FC7) & $313 \mathrm{~m} / \mathrm{s}$ \\
\hline 7 & $385605(2005$ EJ225) & $328 \mathrm{~m} / \mathrm{s}$ \\
\hline 8 & $172974(2005$ YW55) & $342 \mathrm{~m} / \mathrm{s}$ \\
\hline 9 & 96189 Pygmalion (1991 NT3) & $349 \mathrm{~m} / \mathrm{s}$ \\
\hline 10 & $230089(2000$ WP148) & $373 \mathrm{~m} / \mathrm{s}$ \\
\hline
\end{tabular}

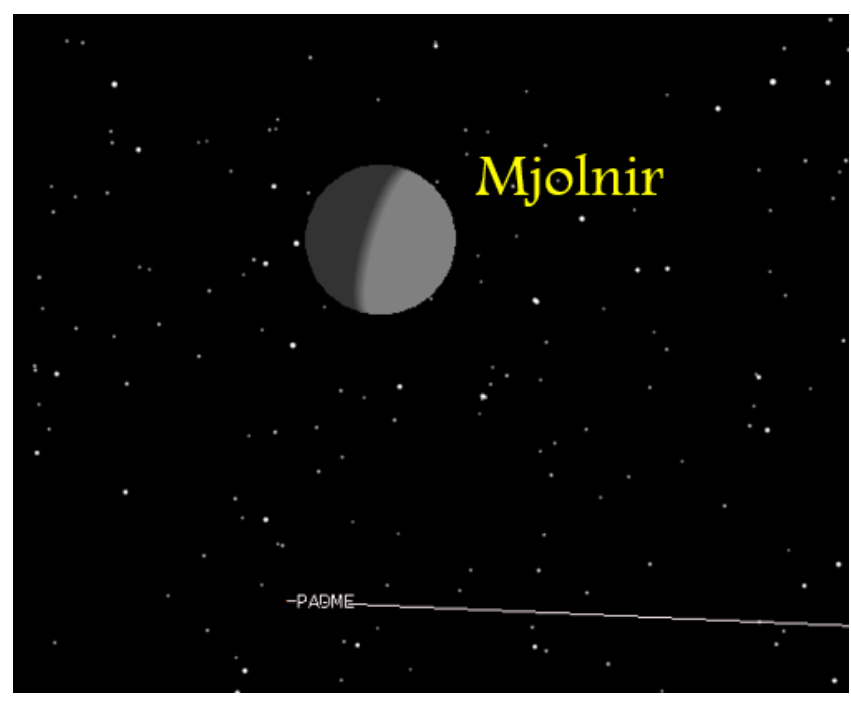

Figure 7. NEO Flyby Opportunity of Mjolnir (Thor's axe-hammer according to Norse mythology). Flyby epoch of April 24, 2024 with a spacecraft flyby speed of $9.5 \mathrm{~km} / \mathrm{s}$ with respect to Mjolnir.

9

American Institute of Aeronautics and Astronautics 
If instead a flyby opportunity to a Mars-crossing asteroid is desired, the PADME spacecraft has several options; the top 15 MCS flyby opportunities are sorted in ascending order by the $\Delta \mathrm{V}$ required and listed in Table 6 . There are 8 opportunities that require less than $250 \mathrm{~m} / \mathrm{s}$ of $\Delta \mathrm{V}$ and are thus within the capability of the PADME spacecraft propulsion system. The MCA that requires the least amount of $\Delta \mathrm{V}$ is 147431 (2003 JA), with $76 \mathrm{~m} / \mathrm{s}$ shown as the required for the transMCA injection (including disposal) given the 2body search performed. Once again, a highfidelity trajectory to 2003 JA (estimated diameter between 900 and 1,800 meters) using STK/Astrogator was calculated. This trajectory is shown in the heliocentric inertial frame (Fig. 8); the spacecraft waits in Mars orbit until September 5, 2023 before performing the injection maneuver, which requires $81 \mathrm{~m} / \mathrm{s}$ in the highfidelity, N-body model. After a 30-month heliocentric coast, the spacecraft performs a flyby of $2003 \mathrm{JA}$ at a speed of $4.34 \mathrm{~km} / \mathrm{s}$, this time targeted to $1 \mathrm{~km}$ from the MCA at closeapproach on November 18, 2024. The spacecraft would remain in heliocentric orbit after the MCA flyby to satisfy disposal requirements of avoiding contact with Mars (without the need to perform a dedicated disposal maneuver since the injection also consists of the disposal maneuver). The total $\Delta \mathrm{V}$ requirement yielded was $958 \mathrm{~m} / \mathrm{s}$, well within the $1,205 \mathrm{~m} / \mathrm{s}$ limit.

Table 6. Top 15 Mars-Crossing Asteroid Flyby Opportunities for the PADME Spacecraft. Opportunities are sorted by $\triangle V$ required for trans-MCA injection from Mars periapsis.

\begin{tabular}{|c|c|c|}
\hline $\begin{array}{c}\text { Opportunity } \\
\text { Number }\end{array}$ & $\begin{array}{c}\text { Mars-Crossing Asteroid } \\
\text { Number (Designation) }\end{array}$ & $\boldsymbol{\Delta}$ V Required for Flyby \\
\hline 1 & 147431 (2003 JA) & $76 \mathrm{~m} / \mathrm{s}$ \\
\hline 2 & $(2014$ AG41) & $109 \mathrm{~m} / \mathrm{s}$ \\
\hline 3 & $(2010$ CV185) & $148 \mathrm{~m} / \mathrm{s}$ \\
\hline 4 & $(2010$ KA58) & $156 \mathrm{~m} / \mathrm{s}$ \\
\hline 5 & $(2012$ BU23) & $158 \mathrm{~m} / \mathrm{s}$ \\
\hline 6 & $(2008$ WX51) & $159 \mathrm{~m} / \mathrm{s}$ \\
\hline 7 & $(2010$ GK68) & $225 \mathrm{~m} / \mathrm{s}$ \\
\hline 8 & $(2012$ KU24) & $238 \mathrm{~m} / \mathrm{s}$ \\
\hline 9 & $(2009$ WP192) & $276 \mathrm{~m} / \mathrm{s}$ \\
\hline 10 & $(2011$ FF29) & $276 \mathrm{~m} / \mathrm{s}$ \\
\hline 11 & $(2010 \mathrm{JH} 100)$ & $282 \mathrm{~m} / \mathrm{s}$ \\
\hline 12 & $(2010$ KD1) & $296 \mathrm{~m} / \mathrm{s}$ \\
\hline 13 & $356948(2012$ VE5) & $295 \mathrm{~m} / \mathrm{s}$ \\
\hline 14 & $(2007$ WA5) & $297 \mathrm{~m} / \mathrm{s}$ \\
\hline 15 & $(2000$ DF8) & \\
\hline
\end{tabular}

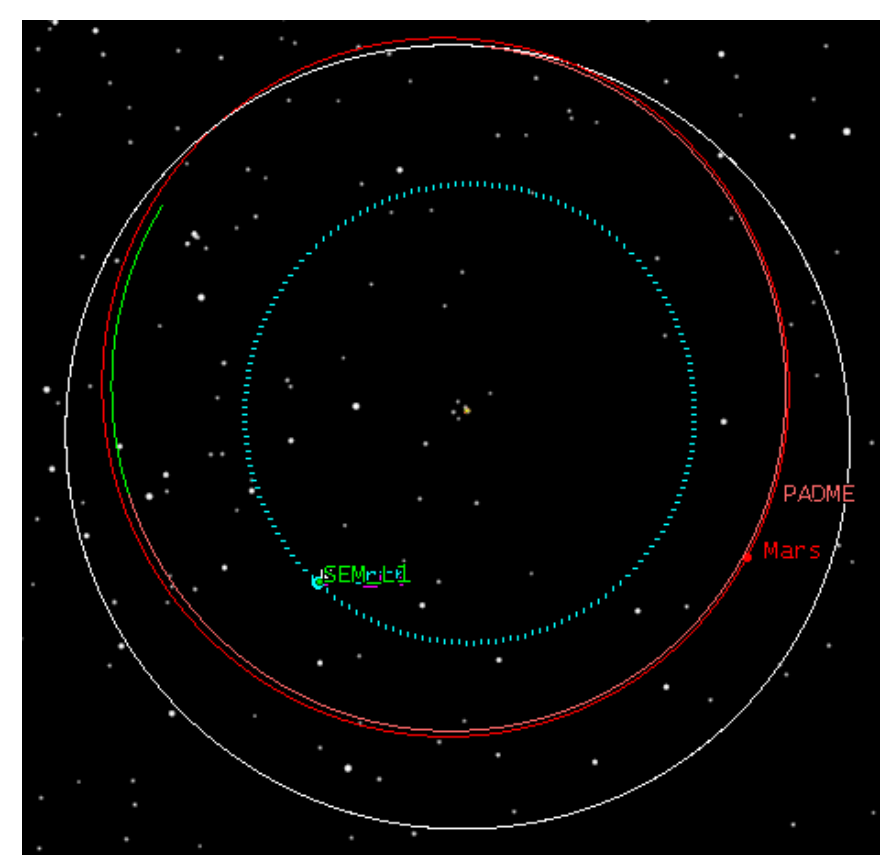

Figure 8. Flyby Opportunity of Mars-Crossing Asteroid. Transfer shown in the heliocentric inertial frame, 147431 (2003 JA) on Nov. 18, 2024. 


\section{E. Future Launch Opportunities to Mars}

Along with the 21-day launch period in August of 2020 for the presented nominal case, launch periods for direct injections occurring in 2022 and 2024 were analyzed as well. The 2024 period was seen to be similar to that of 2020; however, this was not the case for the 2022 period. The major problem for the 2022 injection is the steep arrival declination upon Mars orbit injection. The steep, post-captured Mars orbit is shown in Fig. 9.

By extending the apoapsis to the edge of Mars' sphere of influence, solar gravity "lifts" the trajectory toward Phobos' orbit plane far enough away from Mars such that a tolerable out of plane maneuver, with $250 \mathrm{~m} / \mathrm{s}$ of $\Delta \mathrm{V}$, is performed to enter Phobos' plane and thus reduce the Phobos (and Deimos) flyby speeds to tolerable values (Fig. 10). Specifically, the Phobos flyby speeds will remain the same as those presented in the nominal case (about $1 \mathrm{~km} / \mathrm{s}$ ), while the Deimos flyby speed is increased to $1.39 \mathrm{~km} / \mathrm{s}$ which still satisfies the neutron spectrometer and dust detection flyby speed requirements (Nos. 5 and 6 , Table 1).

Note that the Deimos flybys occur in approximately the same orbit as the Phobos flybys; thus the ALADDIN trajectory design helps reduce the PADME spacecraft's $\Delta \mathrm{V}$ requirement by

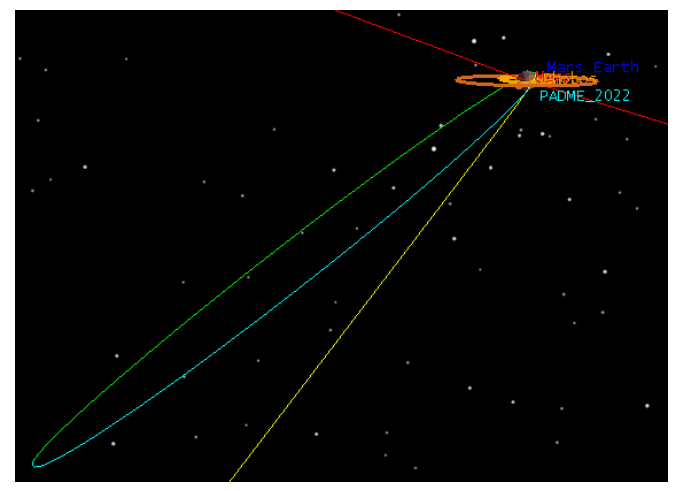

Figure 9. Post-capture Mars orbit for 2022 direct injection opportunity. View of trajectory is edge on Phobos equatorial frame.
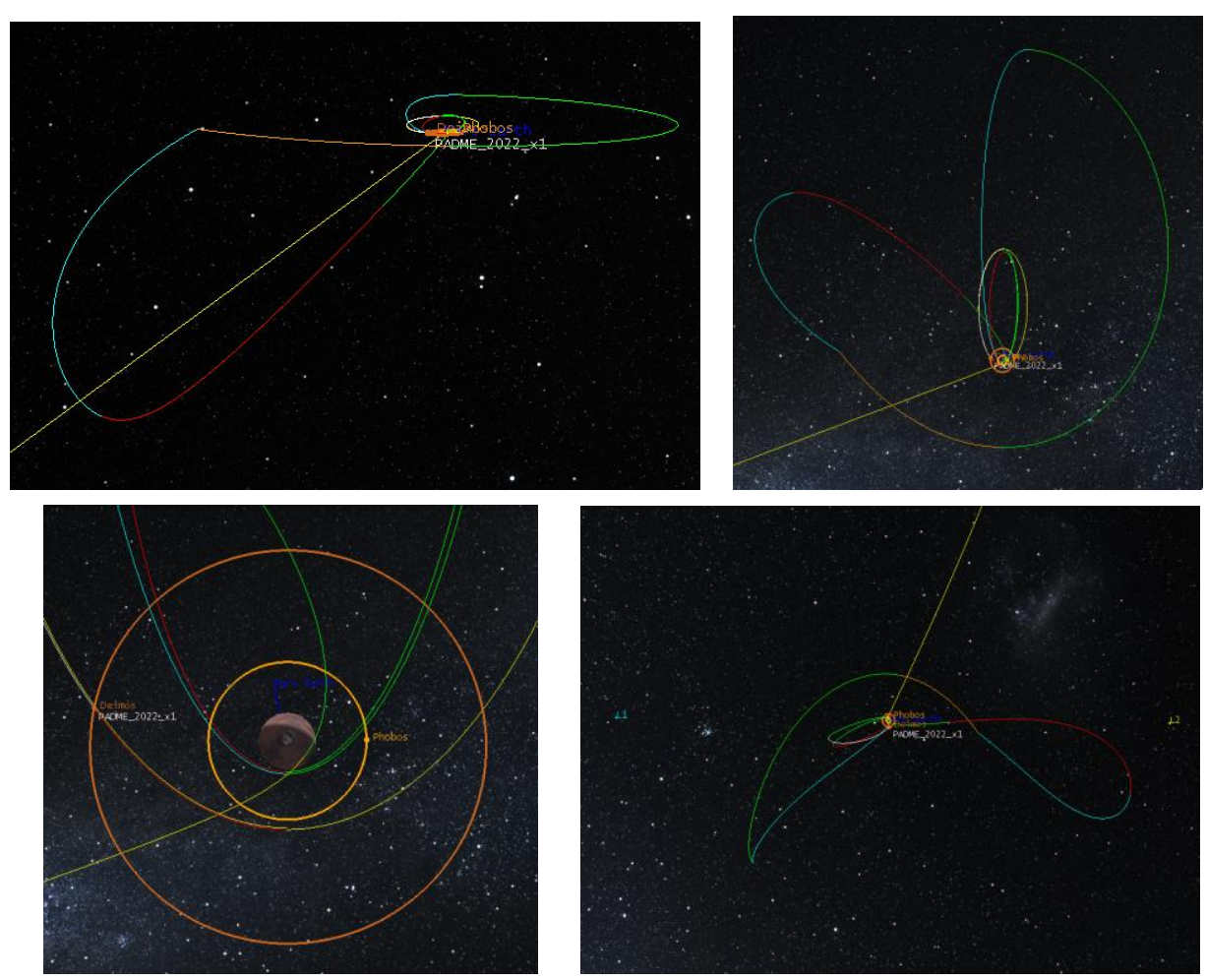

Figure 10. Mars inertial views of the indirect 2022 injection opportunity to the Phobos \& Deimos resonance orbit. Trajectory shown edge-on to the Phobos equatorial plane, (top-left); normal to the Phobos equatorial plane (top-right and bottom-left). Trajectory also shown in the Sun-Mars rotating frame, normal to the Phobos equatorial plane (bottom-right).

eliminating a "gear-switch" and by performing the escape maneuver at a lower Mars periapsis (Fig. 10, bottom-left). Therefore, this 2022 solution becomes marginally feasible as the total $\Delta \mathrm{V}$ required, including disposal to a 100 -year safe orbit (from Mars impact, shown in Fig. 10), was calculated as 1,190 m/s. Optimization of this indirect 2022 solution can further reduce the total $\Delta \mathrm{V}$ requirement, while a heliocentric broken-plane maneuver and/or a solid stage used for the MOI might eliminate the need for the 230-day post-capture trajectory sequence shown in Fig. 10. 


\section{Conclusion}

A trajectory design for the PADME spacecraft has been presented that meets all specified constraints and requirements stated in the Assumptions \& Constraints section (Table 1).

It has been shown that the PADME trajectory was designed in resonance with the orbit of both Phobos and Deimos (Req. No. 3, Table 1), while launched as a primary payload from NASA Kennedy Space Center (Req. No. 1, Table 1) within the $\Delta \mathrm{V}$ budget of $1205 \mathrm{~m} / \mathrm{s}$ (Req. No. 2, Table 1). All flybys have an altitude between 2 and 10 $\mathrm{km}$ above their respective Martian moon (Req. No. 4, Table 1), with at least 8 Phobos and 8 Deimos flybys with a close-approach spacecraft flyby speed less than $1.5 \mathrm{~km} / \mathrm{s}$ (Req. No. 5, Table 1). The dust detection requirement of having two Phobos passes occur at a flyby speed greater than $2 \mathrm{~km} / \mathrm{s}$ is met by any two of Phobos flyby numbers 1-7 (Req. No. 6, Table 1), while this requirement is met for the last two Deimos flybys (> $1 \mathrm{~km} / \mathrm{s})$. The requirement of having (almost) all Phobos and Deimos flyby close-approaches occur in sunlight is met (Req. No. 7 and 9, Table 1), except for the required terminator flyby(s) which is met by Phobos flyby number 4 (Req. No. 8) and Deimos flyby numbers 6, 7, and 8 (Req. No. 10, Table 1). The neutron spectrometer requirement of at least 8 Phobos and 8 Deimos flybys with speed less than $1.5 \mathrm{~km} / \mathrm{s}$ is met by all 16 Phobos and all 9 Deimos flybys. The requirement of having the Earth in view (Req. No. 11, Table 1) during the close-approach of every flyby is satisfied by all 16 Phobos and 9 Deimos flybys. Finally, Phobos flyby numbers 2, 7, 8, and 12 meet the libration science requirements (Req. No. 12 in Table 1), while the requirement of balanced longitude spacing for gravity science (Req. 13, Table 1) is satisfied by all of the Phobos and Deimos flybys.

With the same $\Delta \mathrm{V}$ capability of the lunar LADEE spacecraft, the LADEE-derived PADME spacecraft can reach the required Mars orbit for the analyzed 2020, 2022, and 2024 launch periods, with the ability to conduct valuable and cost-effective science of Phobos and Deimos to help better understand the properties and origin of these Martian moons.

\section{Acknowledgments}

The authors are appreciative of the support given to this effort by the Engineering Directorate and the Technical Library within NASA Ames Research Center at Moffett Field, CA.

\section{References}

${ }^{1}$ Lee, P. et al., "PADME (Phobos and Deimos \& Mars Environment): A Proposed NASA Discovery Mission to Investigate the Two Moons of Mars," presented at the $46^{\text {th }}$ Lunar and Planetary Science Conference, The Woodlands, TX, March 16-20, 2015.

${ }^{2}$ Lee, P. et al., "Phobos and Deimos \& Mars Environment (PADME): A LADEE-Derived Mission to Explore Mars' Moons and the Martian Orbital Environment," presented at the $45^{\text {th }}$ Lunar and Planetary Science Conference, The Woodlands, TX, March 17-21, 2014.

${ }^{3}$ Tolson, R. H., Blanchard, R. C., Daniels, E. F., "Phobos and Deimos Encounter Experiment during the Viking Extended Mission," Journal of Spacecraft \& Rockets, Vol. 13, No. 1, pp. 19-25, 1976.

${ }^{4}$ Diehl, R. E., Adams, M. J., Rinderle Jr., E. A., "Phobos Encounter Trajectory and Maneuver Design," AIAA $16^{\text {th }}$ Aerospace Sciences Meeting, Huntsville, AL, January 16-18, 1978.

${ }^{5}$ Christensen, E. J., Born, G. H., Hildebrand, C. E., Williams, "The mass of Phobos from Viking flybys," Geophysical Research Letters, Vol. 4, No. 12, pp. 555-557, 1977.

${ }^{6}$ Pieters, C., et al., "ALADDIN: Exploration and Sample Return of Phobos and Deimos" $30^{\text {th }}$ Lunar and Planetary Science Conference, 1999. 